\title{
Completely Positive Quasi-Free Maps on the CAR Algebra
}

\author{
David E. Evans \\ Mathematics Institute, University of Copenhagen, 2100 Denmark
}

\begin{abstract}
We construct and study a new class of quasi-free completely positive maps on the $C^{*}$-algebra of the canonical anti-commutation relations.
\end{abstract}

\section{Introduction}

We construct and study quasi-free completely positive maps between algebras of the canonical anti-commutation relations (CAR) induced by contractions at the underlying hilbert space level. We are motivated in part by some analogous results for the Boson case, which however were obtained with less effort using generating functions for example $[7,8,13,14,16,19,20]$. We take as our starting point for the CAR algebra, the theory of quasi-free states, quasi-free automorphisms and the work in $[23,31]$ (see also $[9,20]$ ) on quasi-free completely positive maps which leave the Fock or anti-Fock state invariant.

In the first section, we consider a completely positive contraction on a $C^{*}$ algebra which possesses an invariant state. We study the given map with the aid of the contraction it induces in the GNS Hilbert-space of the invariant state, and show under suitable conditions that a completely positive contraction dominates its spatial part in the GNS decomposition of an invariant KMS state.

In Sect. 2 the theory of quasi-free states on the CAR algebra is reviewed and our notation established. In the third section we construct and develop the theory of a single completely positive quasi-free map. In particular, the main result of Sect. 1 is used to easily identify the pure ones.

In the final section we analyse semigroups of completely positive quasi-free maps on the CAR algebra. In particular, we show that the infinitesimal generator of such a semigroup is bounded at the $C^{*}$-level if and only if it is of trace class at the hilbert space level. This improves the results of [1] (see also [28]) for quasi-free derivations, and a partial result of [9] for quasi-free generators of Fock-type. Some dilation and perturbation questions are also discussed.

We work throughout with CAR algebras built over complex hilbert spaces, but much of our work also holds for those over real spaces. 


\section{Spatiality}

Let $T$ be a completely positive contraction on a $C^{*}$-algebra $A$, which leaves a state $\varphi$ on $A$ invariant. Let $(\pi, H, \xi)$ be the GNS decomposition of $\varphi$.

Then by the Schwarz inequality for a completely positive map there is a well defined contraction $F=F(T)$ say on $H$ such that

$$
F \pi(x) \xi=\pi T(x) \xi, \text { for all } x \text { in } A .
$$

See [24].

In this section we study the relationship between the completely positive map $T$ on $A$ and the completely positive spatial map $F(\cdot) F^{*}$ on $B(H)$. In particular we are interested in showing that

$$
x \rightarrow \pi T(x)-F \pi(x) F^{*}
$$

is completely positive from $A$ into $B(H)$. Such a result has already been obtained in certain circumstances, namely for certain quasi-free completely positive maps on the CCR algebra [20], and for strongly continuous semigroups on a $C^{*}$-algebra which leave invariant a pure state [11].

We will use the definitions and notation of Tomita-Takesaki theory as set out in [34].

Theorem 1.3. Let $T$ and $T^{\prime}$ be completely positive contractions on a $C^{*}$-algebra $A$, having a common invariant separating state $\varphi$ such that

$$
\varphi[T(x) y]=\varphi\left[x T^{\prime}(y)\right], \text { for all } x, y \text { in } A .
$$

Then if $F=F(T)$, the map

$$
x \rightarrow \pi T(x)-F \pi(x) F^{*}
$$

is completely positive from $A$ into $B(H)$.

Proof. Since $\varphi$ is a separating state, it follows from [9, Theorem 4.2] that $T$ and $T^{\prime}$ are spatial, i.e. there exist completely positive normal maps $\hat{T}$ and $\hat{T}^{\prime}$ on the von Neumann algebra $B=\pi(A)^{\prime \prime}$ such that

$$
\hat{T} \pi(x)=\pi T(x), \text { and } \hat{T}^{\prime} \pi(x)=\pi T^{\prime}(x) \text { for all } x \text { in } A .
$$

Then

$$
\langle\hat{T}(a) b \xi, \xi\rangle=\left\langle a \hat{T}^{\prime}(b) \xi, \xi\right\rangle
$$

for all $a, b$ in $B$. The vector state $b \rightarrow\langle b \xi, \xi\rangle: b \in B$, is a faithful normal state on $B$ with modular automorphism group $\left\{\sigma_{t}: t \in \mathbb{R}\right\}$ say, [34]. Then as in $[5,25]$ we see that $\hat{T}$ and $\hat{T}^{\prime}$ commute with the modular automorphism group $\sigma_{\mathbb{R}}$. In fact let $S$ be the closure of the map $b \xi \rightarrow b^{*} \xi ; b \in B$. Then since $\hat{T}$ and $\hat{T}^{\prime}$ are ${ }^{*}$-maps, we see that $F(\hat{T})$ and $F\left(\hat{T}^{\prime}\right)$ commute strongly with $S$. But $F\left(\hat{T}^{\prime}\right)=F(\hat{T})^{*}$ by $(1.4)$ and so $F(\hat{T})$ commutes strongly with both $S$ and $S^{*}$ and hence also with the modular operator $\Delta=\left(S^{*} S\right)^{1 / 2}$. Thus $\hat{T}$ commutes with the modular automorphism group $\sigma_{t}=\Delta^{i t}(\cdot) \Delta^{-i t}$ since $\xi$ is a separating vector for $B$. Similarly $\hat{T}^{\prime} \sigma_{t}=$ $\sigma_{t} \hat{T}^{\prime}$ for all $t$ in $\mathbb{R}$. Let $B_{e}$ denote the $\sigma$-weakly dense *-subalgebra of entire elements of $B$. 
Then

$$
\sigma_{z}(a)^{*}=\sigma_{\bar{z}}\left(a^{*}\right)
$$

and

$$
\left\langle\sigma_{z}(a) \xi, \xi\right\rangle=\langle a \xi, \xi\rangle
$$

for all $a$ in $B_{e}, z$ in $\mathbb{C}$.

Moreover $\hat{T}$ and $\hat{T}^{\prime}$ leave $B_{e}$ invariant and

$$
\hat{T} \sigma_{z}(a)=\sigma_{z} \hat{T}(a), \hat{T}^{\prime} \sigma_{z}(a)=\sigma_{z} \hat{T}^{\prime}(a)
$$

for all $a$ in $B_{e}, z$ in $\mathbb{C}$. We will use the KMS condition in the form:

$$
\langle a b \xi, \xi\rangle=\left\langle b \sigma_{-i}(a) \xi, \xi\right\rangle, \text { for all } a \in B_{e}, b \in B
$$

Then for all $x, y$ and $B_{e}$ we have:

$$
\begin{aligned}
\langle x & \left.x \hat{T}^{\prime}(y) \xi, \hat{T}^{\prime}(y) \xi\right\rangle \\
& =\left\langle\hat{T}^{\prime}\left(y^{*}\right) x^{*} x \hat{T}^{\prime}(y) \xi, \xi\right\rangle \\
& =\left\langle x \hat{T}^{\prime}(y) \sigma_{-i}\left[\hat{T}^{\prime}\left(y^{*}\right) x^{*}\right] \xi, \xi\right\rangle \\
& =\left\langle\sigma_{-i / 2}\left\{\sigma_{i / 2}(x) \sigma_{i / 2}\left[\hat{T}^{\prime}(y)\right] \sigma_{-i / 2}\left[\hat{T}^{\prime}\left(y^{*}\right)\right] \sigma_{-i / 2}\left(x^{*}\right)\right\} \xi, \xi\right\rangle \\
& =\left\langle\sigma_{i / 2}(x) \hat{T}^{\prime}\left(\sigma_{i / 2} y\right) \hat{T}^{\prime}\left[\sigma_{i / 2}(y)^{*}\right] \sigma_{i / 2}(x)^{*} \xi, \xi\right\rangle \\
& \leqq\left\langle\sigma_{i / 2}(x) \hat{T}^{\prime}\left[\sigma_{i / 2}(y) \sigma_{i / 2}(y)^{*}\right] \sigma_{i / 2}(x)^{*} \xi, \xi\right\rangle \\
& =\left\langle\hat{T}^{\prime}\left\{\sigma_{-i / 2}\left[\sigma_{i}(y) y^{*}\right]\right\} \sigma_{-i / 2}\left(x^{*}\right) \sigma_{-i / 2}(x) \xi, \xi\right\rangle \\
& =\left\langle T^{\prime}\left[\sigma_{i}(y) y^{*}\right] x^{*} x \xi, \xi\right\rangle \\
& =\left\langle\sigma_{i}(y) y^{*} \hat{T}\left(x^{*} x\right) \xi, \xi\right\rangle \\
& =\left\langle y^{*} \hat{T}\left(x^{*} x\right) y \xi, \xi\right\rangle
\end{aligned}
$$
by the KadisonSchwarz inequality by (1.5) and (1.8) by (1.6) and (1.7)

The result follows.

The theorem also holds for completely positive maps between different $C^{*}$ algebras with two suitable separating states. The following proposition is an indication of the usefulness of the preceding theorem. Further exploitations of these results will occur in the next section.

Proposition 1.4. Let $\left\{T_{t}: t \in \mathbb{R}^{+}\right\}$be a family of unital linear maps on unital $C^{*}$ algebra $A$ and $\pi$ a non-degenerate representation of $A$ on a Hilbert space $H$. Suppose $\left\{G_{t}: t \in \mathbb{R}^{+}\right\}$is a strongly continuous contraction semigroup on $H$ such that $\lim _{t \rightarrow \infty} G_{t}^{*} G_{t}$ is a projection, $p$, and the map

$$
x \rightarrow \pi T_{t}(x)-G_{t} \pi(x) G_{t}^{*}
$$

is positive from $A$ into $B(H)$. Then

$$
\lim _{t \rightarrow \infty} G_{t}^{*} \pi T_{t}(x) G_{t}
$$

exists in the weak operator topology for all $x$ in $A$, and is equal to $p \pi(x) p$. 
Proof. As $t \rightarrow \infty, G_{t}^{*} G_{t}$ converges strongly to a projection $p$ [33]. Let $J_{t}$ denote the positive map from $A$ into $B(H)$ given in (1.9). Then for all $x \in A^{+}, t \in \mathbb{R}^{+}, \xi \in H$ we have

$$
\begin{aligned}
\left\langle G_{t}^{*} J_{t}(x) G_{t} \xi, \xi\right\rangle & \leqq\|x\|\left\langle G_{t}^{*} J_{t}(1) G_{t} \xi, \xi\right\rangle \\
& =\|x\|\left\langle\left(G_{t}^{*} G_{t}-G_{t}^{*} G_{t} G_{t}^{*} G_{t}\right) \xi, \xi\right\rangle \\
& \rightarrow 0 \text { as } t \rightarrow \infty .
\end{aligned}
$$

Hence by linearity we see that

$$
\lim _{t \rightarrow \infty} G_{t}^{*} \pi T_{t}(x) G_{t}=p \pi(x) p
$$

for all $x$ in $A$.

\section{The CAR Algebra}

Here we record what we need about quasi-free states on the CAR algebra, details of which can be found in $[1,2,4,12,21,27,30,32]$.

If $H$ is a hilbert space, the CAR algebra over $H, A(H)$ is the $C^{*}$-algebra generated by elements $\{a(f): f \in H\}$ where $a$ is a conjugate-linear map from $H$ into $A(H)$ satisfying the canonical anti-commutation relations:

$$
\begin{aligned}
& a(f) a(g)+a(g) a(f)=0 \\
& a^{*}(f) a(g)+a(g) a^{*}(f)=\langle f, g\rangle 1
\end{aligned}
$$

for all $f, g$ in $H$, and where $a^{*}(f)=a(f)^{*}$.

Fock space $F(H)$ is defined to be $\oplus \Lambda^{n} H$, where $\Lambda^{0} H=\mathbb{C}$ with unit vector $\Omega$, and $\Lambda^{n} H$ for $n \geqq 1$ is the $n$-fold anti-symmetric tensor product of $H$. If $T$ is a contraction between hilbert spaces $H$ and $K, F(T)$ will denote the contraction determined by

$$
F(T) g_{1} \Lambda \ldots \Lambda g_{n}=T g_{1} \Lambda \ldots \Lambda T g_{n}, \quad g_{i} \in H .
$$

The formula

$$
\pi_{0} a^{*}(f) g_{1} \Lambda \ldots \Lambda g_{n}=f \Lambda g_{1} \Lambda \ldots \Lambda g_{n}, f, g_{i} \in H
$$

defines an irreducible representation of the CAR algebra $A(H)$ on $F(H)$ called the Fock representation. Further representations of $A(H)$ can be obtained as follows. If $H$ is a hilbert space, $\bar{H}$ will denote its conjugate hilbert space with the canonical conjugate-linear isometry $f \rightarrow \bar{f}$ of $H$ onto $\bar{H}$. For an operator $R$ on $H$ with $0 \leqq R \leqq 1$, take positive operators $\alpha, \beta$ on $H$ such that $\alpha^{2}+\beta^{2}=1$ and $\beta^{2}=R$.

Let $\Gamma=F(-1)$ be the self adjoint unitary on $F(H)$ such that $\Gamma \Omega=\Omega$ and $\Gamma a(f)=-a(f) \Gamma$ for all $f$ in $H$. Then

$$
\pi_{R}^{\prime}: a(f) \rightarrow \pi_{0} a(\alpha f) \otimes \Gamma+1 \otimes \pi_{0} a(\overline{\beta f})^{*} ; \quad f \in H
$$

defines a representation $\pi_{R}^{\prime}$ of the CAR algebra $A(H)$ on $F(H) \otimes F(\bar{H})$. We define 
the quasi-free state $\omega_{R}$ on $A(H)$ by

$$
\omega_{R}(x)=\left\langle\pi_{R}^{\prime}(x) \Omega \otimes \Omega, \Omega \otimes \Omega\right\rangle ; x \in A(H)
$$

so that

$$
\begin{aligned}
\omega_{R} & {\left[a^{*}\left(f_{n}\right) \ldots a^{*}\left(f_{1}\right) a\left(g_{1}\right) \ldots a\left(g_{m}\right)\right] } \\
& =\operatorname{det}\left[\left\langle R f_{i}, g_{j}\right\rangle\right] \delta_{n m}
\end{aligned}
$$

We denote the GNS decomposition of $\omega_{R}$ by $\left(\pi_{R}, F_{R}(H), \Omega_{R}\right)$, so that $F_{R}(H)$ can be identified with $\left[\pi_{R}^{\prime} A(H) \Omega \otimes \Omega\right]^{-}, \Omega_{R}$ with $\Omega \otimes \Omega$ and $\pi_{R}$ with a subrepresentation of $\pi_{R}^{\prime}$.

We can decompose $H$ as $H_{1} \oplus H_{2}$ where $H_{1}=\operatorname{Ker}[|1-2 R|-1]$ and $H_{2}=H \ominus H_{1}$. Then $R H_{i} \subseteq H_{i}$ for $i=1,2$, and if $R_{i}=\left.R\right|_{H_{i}}$ we have $R_{1}^{2}=R_{1}$. The quasi-free state $\omega_{R}$ is said to have no Fock part if $H_{1}=0$, and to be completely Fock if $H_{2}=0$, or equivalently if $R^{2}=R$.

If $R$ has no Fock part, the representation $\pi_{R}^{\prime}$ is already cyclic with cyclic vector $\Omega_{R}$. Moreover in this situation, the state $\omega_{R}$ is KMS at inverse temperature $\beta \neq \infty$, for the quasi-free group of automorphisms $A\left(e^{i h t}\right)$ where the self-adjoint operator $h$ on $H$ is given by $\operatorname{coth}(\beta h / 2)=1-2 R$.

\section{Quasi-Free Completely Positive Maps}

Let $T: H \rightarrow K$ be a contraction between hilbert spaces $H$ and $K$. We wish to construct in a quasi-free fashion completely positive maps from $A(H)$ into $A(K)$ extending $T$ in the sense that $a(f)$ must be mapped onto $a(T f)$. Moreover we with this construction to be functorial and to reduce to the study of quasi-free states for the case $T=0$, and to quasi-free *-homomorphisms for isometric $T$.

Thus if $T$ is an isometry we define $A(T)$ to be the unique *-homomorphism from $A(H)$ into $A(K)$ such that $A(T) a(f)=a(T f)$, for all $f$ in $H$.

Next we show that projections at the hilbert space level will give rise to conditional expectations at the $C^{*}$-level. Thus let $H$ and $K$ be hilbert spaces, and $R$ an operator on $H$ with $0 \leqq R \leqq 1$. Consider the *-homomorphism $\pi$ of $A(K \oplus H)$ into $A(K) \otimes B\left(F_{R}(H)\right)$ given by

$$
\pi a(k \oplus h)=a(k) \otimes \Gamma_{R}+1 \otimes \pi_{R} a(h), \quad k \oplus h \in K \oplus H
$$

where $\Gamma_{R}$ is the restriction of $\Gamma \otimes \Gamma$ to $F_{R}(H)$. We can then define a projection $N$ from $A(K \oplus H)$ onto $A(K)$ by

$$
x \rightarrow 1 \otimes \tilde{\omega}_{R} \pi(x) ; \quad x \in A(K \oplus H)
$$

where $\tilde{\omega}_{R}$ is the vector state $\Omega_{R} \otimes \bar{\Omega}_{R}$ on $B\left(F_{R}(H)\right)$. Then for any operator $S$ on $K$ with $0 \leqq S \leqq 1$, we have

$$
\omega_{S \oplus R}=\omega_{S} \circ N
$$

Note the existence of a conditional expectation $N$ satisfying (3.2) follows from [35] for case when $R$ and $S$ have no Fock parts, and then the general case follows if one uses a limiting argument. 
Now let $T: H \rightarrow K$ be an arbitrary contraction between hilbert spaces $H$ and $K$. Then if $U$ denotes the unitary

$$
\left[\begin{array}{cc}
-\left(1-T T^{*}\right)^{1 / 2} & T \\
T^{*} & \left(1-T^{*} T\right)^{1 / 2}
\end{array}\right] \quad \text { on } K \oplus H
$$

and $W_{1}^{\prime}$ and $W_{2}$ are the isometries $h \rightarrow(0, h), h \in H ; k \rightarrow(k, 0), k \in K$ respectively, one has the unitary dilation $T=W_{2}^{*} U W_{1}^{\prime}$ for $T$ [22]. In particular $T=W_{2}^{*} W_{1}$ is a decomposition of $T$ into a co-isometry and an isometry where

$$
W_{1} h=U W_{1}^{\prime} h=T h \oplus\left(1-T^{*} T\right)^{1 / 2} h ; \quad h \in H .
$$

If $R$ is an operator on $H$ with $0 \leqq R \leqq 1$, we define the completely positive map $A_{R}(T)$ by composition:

$$
A_{R}(T)=N \circ A\left(W_{1}\right)
$$

where $N$ is the conditional expectation in (3.1) of $A(K \oplus H)$ onto $A(K)$. Taking $R=0$, we recover the quasi-free completely positive maps constructed in $[23,31]$, see also $[9,20]$.

In the first place note that $A_{R}(T)$ maps the quasi-free states of $A(K)$ into those of $A(H)$. Let $S$ be an operator on $K$ with $0 \leqq S \leqq 1$. Then

$$
\begin{aligned}
\omega_{S} A_{R}(T) & =\omega_{S} N A\left(W_{1}\right) \\
& =\omega_{S \oplus R} A\left(W_{1}\right) \\
& =\omega_{Q}
\end{aligned}
$$

where $Q=W_{1}^{*}(S \oplus R) W_{1}=T^{*} S T+\left(1-T^{*} T\right)^{1 / 2} R\left(1-T^{*} T\right)^{1 / 2}$. (In particular, $\omega_{1} A_{0}(T)=\omega_{T^{*} T}$, in accordance with [23]).

Thus if $T R=S T$ we have

$$
\omega_{S} A_{R}(T)=\omega_{R} .
$$

In fact even more is true:

$$
\left.\omega_{S}\left[A_{R}(T)(x)\right] y\right]=\omega_{R}\left[x A_{S}\left(T^{*}\right)(y)\right]
$$

for all $x$ in $A(H), y$ in $A(K)$, since

$$
\begin{aligned}
\omega_{S} & {\left[A_{R}(T)(x) y\right]=\omega_{S}\left[N A\left(W_{1}\right)(x) y\right] } \\
& =\omega_{S} N\left[A\left(W_{1}\right) x A\left(W_{2}\right)(y)\right] \\
& =\omega_{S \oplus R}\left[A(U) A\left(W_{1}^{\prime}\right)(x) A\left(W_{2}\right)(y)\right] \\
& \left.=\omega_{S \oplus R} A\left(W_{1}^{\prime}\right)(x) A\left(U^{-1}\right) A\left(W_{2}\right)(y)\right] \quad \text { as }[U, S \oplus R]=0 \\
& =\omega_{R}\left[x A_{S}\left(T^{*}\right)(y)\right] .
\end{aligned}
$$

Note that if (3.3) holds then it must be the case that $T R=S T$, by considering $x=a(h), y=a(k), h \in H, k \in K$. Moreover if $R$ and $S$ have no Fock part, and if $\omega_{S}\left[A_{R}(T)(x) y\right]=\omega_{R}[x \varphi(y)] ; x \in A(H), y \in A(K)$ for some unital positive map $\varphi$ from $A(K)$ into $A(H)$, then we see from the proof of theorem 1.3 that $T R=S T$.

Now let $T$ be an arbitrary contraction between Hilbert spaces $H$ and $K$ and $R$ an operator on $H, 0 \leqq R \leqq 1$. Then if $D=\left(1-T^{*} T\right)^{1 / 2}$ and $f_{1}, \ldots, f_{n}$, are ele- 
ments of $H$, we have from the definitions:

$$
\begin{aligned}
A_{R}(T)\left[a^{\#}\left(f_{1}\right) \ldots a^{\#}\left(f_{n}\right)\right] \\
=\Sigma \varepsilon a^{\#}\left(T f_{1}\right) \ldots a^{\#}\left(\hat{T} f_{i_{1}}\right) \ldots a^{\#}\left(\hat{T} f_{i_{p}}\right) \ldots a^{\#}\left(T f_{n}\right) \\
\quad \cdot \omega_{R}\left[a^{\#}\left(D f_{i_{1}}\right) \ldots a^{\#}\left(D f_{i_{p}}\right)\right]
\end{aligned}
$$

Here $a^{\#}(f)$ denotes either $a(f)$ or $a^{*}(f)$ but no normal ordering is necessary. The summation extends over all subsets $\sigma=\left\{i_{1}, \ldots, i_{p}\right\}$ of $\{1, \ldots, m\}$ in increasing order; and if $\sigma^{\prime}=\left\{j_{1}, \ldots, j_{n-p}\right\}$ is the complement of $\sigma$ in $\{1, \ldots, n\}$, in increasing order, the $\varepsilon$ is the signature of the permutation $(1, \ldots, n) \rightarrow\left(\sigma, \sigma^{\prime}\right)$. Also over a term $a^{\#}(f)$ means that this factor is omitted. $h \in H\}$ :

In particular $A_{R}(T)$ is a homomorphism on the algebra generated by $\{a(h)$ :

$$
A_{R}(T)\left[a\left(f_{1}\right) \ldots a\left(f_{n}\right)\right]=a\left(T f_{1}\right) \ldots a\left(T f_{n}\right) .
$$

If $\left[R, T^{*} T\right]=0$, then this expression simplifies (3.4) somewhat. Assume for the remainder of this section that there exists an operator $S$ on $K$ with $0 \leqq S \leqq 1$, and $T R=S T$. Then even greater simplifications take place as we shall discover.

If $R=0, S=0$, then we have [23]:

$$
\begin{aligned}
& A_{0}(T)\left[a^{*}\left(f_{1}\right) \ldots a^{*}\left(f_{r}\right) a\left(f_{r+1}\right) \ldots a\left(f_{n}\right)\right] \\
& \quad=a^{*}\left(T f_{1}\right) \ldots a^{*}\left(T f_{r}\right) a\left(T f_{r+1}\right) \ldots a\left(T f_{n}\right) .
\end{aligned}
$$

Also if $R=1, S=1$, then

$$
\begin{aligned}
& A_{1}(T)\left[a\left(f_{1}\right) \ldots a\left(f_{r}\right) a^{*}\left(f_{r+1}\right) \ldots a^{*}\left(f_{n}\right)\right] \\
& \left.\quad=a\left(T f_{1}\right) \ldots a\left(T f_{r}\right) a^{*}\left(T f_{r+1}\right) \ldots a^{*}\left(T f_{n}\right)\right]
\end{aligned}
$$

However the fock functor $A_{0}$ does not have a simple expression on Wick monomials, normally ordered with respect to the anti-Fock state $\omega_{1}$, and vice-versa. It would thus seem useful to introduce normal ordering with respect to arbitrary quasi-free states.

Now if $g_{1}, \ldots, g_{n}$ and $f_{1}, \ldots, f_{m}$ are elements of $H$, we have

$$
\begin{aligned}
a\left(g_{n}\right) \ldots a\left(g_{1}\right) a^{*}\left(f_{1}\right) \ldots a^{*}\left(f_{m}\right) \\
=\sum \varepsilon a^{*}\left(f_{1}\right) \ldots a^{*}\left(\hat{f}_{i_{1}}\right) \ldots a^{*}\left(\hat{f}_{i_{p}}\right) \ldots a^{*}\left(f_{m}\right) \\
\quad \cdot a\left(g_{n}\right) \ldots a\left(\hat{g}_{j_{p}}\right) \ldots a\left(\hat{g}_{j_{1}}\right) \ldots a\left(g_{1}\right) \\
\quad \cdot \operatorname{det}\left[\left\langle f_{i_{r}}, g_{j_{s}}\right\rangle\right]_{r, s}
\end{aligned}
$$

where the summation extends over all subsets $\sigma=\left\{i_{1}, \ldots, i_{p}\right\}$ (respectively $\rho=$ $\left\{j_{1}, \ldots, j_{p}\right\}$ ) of $\{1,2, \ldots, m\}$ (respectively $\left.\{1,2, \ldots, n\}\right)$ in increasing order, and if $\sigma^{\prime}=\left\{i_{1}^{\prime} \ldots i_{m-p}^{\prime}\right\}$ (respectively $\rho^{\prime}=\left\{j_{1}^{\prime} \ldots, j_{n-\rho}^{\prime}\right\}$ in increasing order is the complement of $\sigma$ (respectively $\rho$ ) in $\{1,2, \ldots, m\}$ (respectively $\{1,2, \ldots, n\}$ ) then $\varepsilon$ is the product of $(-1)^{(m-p)(n-p)}$ and the signatures of permutations 
state $\omega_{R}$ can then be defined by:

$$
\begin{aligned}
: a^{*}\left(f_{1}\right) \ldots a^{*}\left(f_{m}\right) a\left(g_{n}\right) \ldots a\left(g_{1}\right):_{R} \\
=\sum \varepsilon a^{*}\left(f_{1}\right) \ldots a^{*}\left(\hat{f}_{i_{1}}\right) \ldots a^{*}\left(\hat{f}_{i_{p}}\right) \ldots a^{*}\left(f_{m}\right) \\
\quad \cdot a\left(g_{n}\right) \ldots a\left(\hat{g}_{j_{p}}\right) \ldots a\left(\hat{g}_{j_{1}}\right) \ldots a\left(g_{1}\right) \\
\quad \cdot \operatorname{det}\left[\omega_{R}\left(a^{*}\left(f_{i_{r}}\right) a\left(g_{j_{s}}\right)\right)\right]
\end{aligned}
$$

where the summation is as described for (3.6).

We simplify the notation even further so that when we write $: a^{\#}\left(h_{1}\right) \ldots a^{\#}\left(h_{n}\right):_{R}$ for some vectors $h_{1}, \ldots, h_{n}$ in $H$, it is always implicit that inside the dots, all the creation operators are to the left, and the annihilation operators to the right.

Then if $T$ is a contraction between hilbert spaces $H$ and $K$ which intertwines with operators $R$ and $S$ on $H$ and $K$ respectively, with $0 \leqq R \leqq 1,0 \leqq S \leqq 1$, it is shown in the appendix that:

$$
\begin{gathered}
A_{R}(T)\left[: a^{\#}\left(h_{1}\right) \ldots a^{\#}\left(h_{n}\right):_{R}\right] \\
\quad=: a^{\#}\left(T h_{1}\right) \ldots a^{\#}\left(T h_{n}\right):_{S}
\end{gathered}
$$

for all $h_{1}, \ldots, h_{n}$ in $H$. (However recall from the definition that $A_{R}(T)$ in no way depends on $S$ ).

From this we can conclude that if $T^{\prime}$ is another contraction from $K$ into another Hilbert space $L$, and that if there exists an operator $Q$ on $L$, with $0 \leqq Q \leqq 1$, and $T^{\prime} S=Q T^{\prime}$, then

$$
A_{S}\left(T^{\prime}\right) A_{R}(T)=A_{R}\left(T^{\prime} T\right) .
$$

We now study the contraction $F_{R, S}(T)$ from $F_{R}(H)$ into $F_{S}(K)$ given by:

$$
F_{R, S}(T) \pi_{R}(x) \Omega_{R}=\pi_{S} A_{R}(T)(x) \Omega_{S} ; \quad x \in A(H)
$$

If $H=K$ and $R=S$, we write $F_{R}(T)$ for $F_{R, R}(T)$. We claim that:

$$
F_{R, S}(T) \text { is the restriction of } F(T) \otimes F(\bar{T}) \text { to } F_{R}(H)
$$

where $\bar{T}$ is the bounded operator $x \rightarrow \overline{T x}$ on $\bar{H}$. If $T$ is an isometry then (3.10) can be verified by a direct calculation involving the very definitions. But from (3.3) we have $F_{R, S}(T)^{*}=F_{S, R}\left(T^{*}\right)$, and so from $F\left(T^{*}\right) \otimes F\left(\overline{T^{*}}\right)=[F(T) \otimes F(\bar{T})]^{*}$ it will follow that (3.10) also holds when $T$ is a co-isometry. Hence the decomposition $T=W_{2}^{*} W_{1}$ and the definition of $A_{R}(T)$ allows us to deduce (3.9) in the general case. (Note that $S \oplus R W_{1}=W_{1} R$ and $S \oplus R W_{2}=W_{2} S$ ). It is interesting to note that our knowledge of $T \rightarrow A_{R}(T)$ being functorial is not required here. Thus (3.9) and (3.10) can be used to prove the functorial property (3.8) in the first place for $R$ and $S$ which have no Fock part in which case $\Omega_{R}$ and $\Omega_{S}$ are separating, and secondly for arbitrary $R$ and $S$ by a limiting argument which will be described in our next result, (c.f. [31]).

$$
\begin{aligned}
& \text { Let } \\
& R_{n}=1 / n+(1-2 / n) R
\end{aligned}
$$

and

$$
S_{n}=1 / n+(1-2 / n) S .
$$


Then $R_{n}$ and $S_{n}$ have no Fock parts, $T R_{n}=S_{n} T$, and $R_{n} \rightarrow R, S_{n} \rightarrow S$ as $n \rightarrow \infty$. Thus we conclude from Theorem 1.3 that

$$
x \rightarrow \pi_{S} A_{R}(T)(x)-F \pi_{R}(x) F^{*}
$$

is completely positive from $A$ into $B\left(F_{S}(K)\right)$, where $F=F_{R, S}(T)$. In what follows we will often dispense with the symbols $\pi_{R}$ and $\pi_{S}$ when taking representations.

If $T$ is a co-isometry we have

$$
A_{R}(T)(1)-F_{R, S}(T) 1 F_{R, S}(T)^{*}=0
$$

and so from the preceding paragraph, we have

$$
A_{R}(T)=F(\cdot) F^{*} \text {. }
$$

In particular $A_{R}(T)$ has an ultraweak extension $\tilde{A}_{R}(T)$ from $\pi_{R}(A(H))^{\prime \prime}$ into $\pi_{S}(A(K))^{\prime \prime}$. If $R$ and $S$ have no Fock parts it follows from (3.10) and (3.12) that

$$
A_{R}(T)(x)=A_{0}(T) \otimes \tilde{A}_{0}(T)(x) ; \quad x \in A(H)
$$

Note however that simply by considering $x=a(h), h \in H$, that (3.13) fails if $T$ is not co-isometric. This should be contrasted with the CCR situation (see [20]) where the analogous result is easily seen to hold for any contraction $T$.

Conversely suppose (3.12) holds for some contraction $T$. Then $1=F_{R, S}(T) \times$ $F_{R, S}\left(T^{*}\right)=F_{S}\left(T T^{*}\right)$, and by considering the action on $\pi_{S}\left(a^{*}(h)\right)$ it follows that $T$ is a co-isometry.

The complete positivity of (3.11) can also be used to easily determine when $A_{R}(T)$ is pure in the sense of [3,14 Definition 3.2]. If $A_{R}(T)$ is pure then we must have

$$
\lambda A_{R}(T)=F(\cdot) F^{*}
$$

for some $\lambda \geqq 0$. In particular we see

$$
\lambda \Omega_{S}=\lambda A_{R}(T)(1) \Omega_{S}=F_{R, S}(T) 1 F_{R, S}(T)^{*} \Omega_{S}=\Omega_{S},
$$

and so $\lambda=1$. Thus from the preceding $T$ must be a co-isometry. Then from (3.12) and [3] we see that if $T$ is a co-isometry then $A_{R}(T)$ is pure if and only if the representation $\pi_{R}$ is irreducible i.e. if and only if $R$ is a projection.

We summarize these results as follows. Note that (iii) is in accordance with [14] for the CCR algebra, which however has shown by an entirely different method.

Theorem 3.1. Let $T$ be a contraction between hilbert spaces $H$ and $K$, with $R, S$ operators on $H$ and $K$ respectively such that $0 \leqq R \leqq 1,0 \leqq S \leqq 1$ and $T R=S T$. Then there exists an unique completely positive unital map $A_{R}(T)$ from $A(H)$ into $A(K)$ such that

$$
\mathrm{A}_{R}(T): a^{\#}\left(f_{1}\right) \ldots a^{\#}\left(f_{n}\right):_{R}=: a^{\#}\left(T f_{1}\right) \ldots a^{\#}\left(T f_{n}\right):_{S}
$$

for all $f_{1}, \ldots, f_{n}$ in $H$.

Moreover:

(i) $\omega_{S}\left[A_{R}(T)(x) y\right]=\omega_{R}\left[x A_{S}\left(T^{*}\right) y\right], x \in A(H), y \in A(K)$ 
(ii) The map $x \rightarrow \pi_{S} A_{R}(T)(x)-F \pi_{R}(x) F^{*}$ is completely positive from $A(H)$ into $B\left(F_{S}(K)\right)$, and where $F$ is the contraction from $F_{R}(H)$ into $F_{S}(K)$ given by $\pi_{R}(x) \Omega_{R} \rightarrow \pi_{S} A_{R}(T)(x) \Omega_{S}$. Also $\pi_{S} A_{R}(T)(x)=F \pi_{R}(x) F^{*}$, for all $x$ in $A(H)$ if and only if $T$ is a co-isometry, and $A_{R}(T)$ is a homomorphism if and only if $T$ is an isometry.

(iii) $A_{R}(T)$ is pure if and only if $T T^{*}=1$ and $R^{2}=R$, i.e. $A_{R}(T)$ is a conditional expectation with respect to a Fock state.

\section{Semigroups of Quasi-Free Completely Positive Maps}

Let $\left\{T_{t}: t \geqq 0\right\}$ be a strongly continuous contraction semigroup on a Hilbert space $H$, which commutes with an operator $R$ on $H, 0 \leqq R \leqq 1$. We now study the dynamical semigroup $A_{R}\left(T_{t}\right)$ on $A(H)$.

Suppose, c.f. [33], we know that as $t \rightarrow \infty, T_{t}^{*} T_{t}$ converges strongly to some projection $e$ on $H$, which reduces the semigroup $T_{t}$, and is such that $T_{t}$ is isometric on $e H$, and converges strongly to zero on $(1-e) H$. Then as $t \rightarrow \infty, F_{R}\left(T_{t}\right)^{*} F_{R}\left(T_{t}\right)=$ $F_{R}\left(T_{t}^{*} T_{t}\right)$ converges strongly to $F_{R}(e)$. Thus by Proposition 1.4 we see that as $t \rightarrow \infty$ :

$$
\begin{aligned}
F_{R}\left(T_{t}\right) A_{R}\left(T_{t}\right)(\cdot) F_{R}\left(T_{t}^{*}\right) & \rightarrow F_{R}(e)(\cdot) F_{R}(e) \\
& =A_{R}(e)(\cdot)
\end{aligned}
$$

which can be interpreted as a conditional expectation onto the hamiltonian part of the system.

To dilate the semigroup $A_{R}\left(T_{t}\right)$, first take a minimal unitary dilation $\left(U_{t}, K, V\right)$ of the semigroup $T_{t}$ [33], so that $V$ is an isometric embedding of $H$ as a subspace of a hilbert space $K$, and $U_{t}$ is a strongly continuous unitary group on $K$ satisfying

$$
K=\mathrm{v}\left\{U_{t} V f: t \in \mathbb{R}, f \in H\right\}
$$

and

$$
T_{t}=V^{*} U_{t} V, t \geqq 0 .
$$

Let $\hat{T}_{t}=T_{t}$ if $t \geqq 0$, and $\hat{T}_{t}=T_{-t}^{*}$ if $t \leqq 0$. Then

$$
\begin{aligned}
(t, s) & \rightarrow\left(1-R^{2}\right)^{1 / 2} \hat{T}_{s-t}\left(1-R^{2}\right)^{1 / 2} \\
& =\hat{T}_{s-t}-R \hat{T}_{s-t} R
\end{aligned}
$$

is positive definite on $\mathbb{R} \times \mathbb{R}$. Hence by [20, Theorem 1.12] there exists an unique contraction $S$ on $K$ such that $S U_{t} V=U_{t} V R$, for all $t$ in $\mathbb{R}$. It is easily verified that $S V=V R,\left[S, U_{t}\right]=0$, and that $S$ is positive. Thus we see from the preceding theory that the semigroup of completely positive maps $A_{R}\left(T_{t}\right)$ on $A(H)$ can be dilated to a group of *-automorphisms $A\left(U_{t}\right)$ on $A(K)$ :

$$
A_{R}\left(T_{t}\right)=A_{S}\left(V^{*}\right) A\left(U_{t}\right) A(V) ; \quad \text { for all } t \geqq 0 .
$$

Such dilations have been widely studied in the analogous CCR case $[7,8,16$, 20]. Other types of dilations can also be obtained as in [20], based either on Cooper's dilation [6] or on the co-isometric dilation of [10, Theorem 16.2] rather than based on St. Nagy's dilation [33] as we have done above. See also [14]. 
Again let $\left\{T_{t}: t \geqq 0\right\}$ be a strongly continuous contraction semigroup on a Hilbert space $H$, which commutes with an operator $R$ on $H, 0 \leqq R \leqq 1$. If $B$ denotes the infinitesimal generator of the semigroup $T_{t}$, let $d A_{R}(B)$ denote the infinitesimal generator of the strongly continuous contraction semigroup $A_{R}\left(e^{B t}\right)$ at the $C^{*}$-level. Consider now the problem of characterising for which $B$ the induced semigroup $A_{R}\left(e^{B t}\right)$ is norm continuous. If $A_{R}\left(e^{B t}\right)$ is norm continuous, then $B$ is certainly bounded since $A_{R}\left(e^{B t}\right) a(f)=a\left(e^{B t} f\right)$. Moreover a bounded operator $B$ on $H$ gives rise to a contraction semigroup $\left\{e^{B t}: t \geqq 0\right\}$ if and only if $B$ is dissipative; i.e. $r e(B) \leqq 0$. It was shown in [1], (see also [28]) that if $B$ is skew-adjoint or equivalently if $e^{B t}$ is a unitary group, then $A\left(e^{B t}\right)$ is a norm conti-

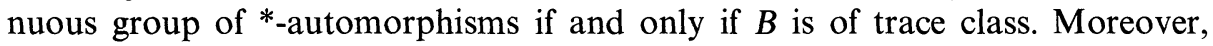
it was also shown in [9] that a dissipative trace class operator $B$ on $H$ gives rise to a norm continuous semigroup $A_{0}\left(e^{B t}\right)$, here 0 refers to the Fock-functor of $[23,31])$. These results will be improved in Theorem 4.1 .

However, first consider a bounded dissipative operator $B$ on $H$ which commutes with an operator $R$ on $H, 0 \leqq R \leqq 1$. Then from (3.7) we see that the *algebra $A^{1}(H)$ generated by $\{a(h): h \in H\}$ is contained in the domain of $d A_{R}(B)$ and

$$
\begin{aligned}
& d A_{R}(B)\left[: a^{\#}\left(h_{1}\right) \ldots a^{\#}\left(h_{n}\right):_{R}\right] \\
& \quad=\sum_{i=1}^{n}: \prod_{j=1}^{i-1} a^{\#}\left(h_{j}\right) a^{\#}\left(B h_{i}\right) \prod_{j=i+1}^{n} a^{\#}\left(h_{j}\right):_{R}
\end{aligned}
$$

for all $h_{1}, \ldots, h_{n}$ in $H$.

Thus if $B_{1}$ and $B_{2}$ are dissipative operators commuting with $R$, then

$$
d A_{R}\left(B_{1}+B_{2}\right)(x)=d A_{R}\left(B_{1}\right)(x)+d A_{R}\left(B_{2}\right)(x)
$$

for all $x$ in $A^{1}(H)$.

Theorem 4.1. Let $\left\{e^{B t}: t \geqq 0\right\}$ be a strongly continuous contraction semigroup on a Hilbert space $H$, which commutes with an operator $R$ on $H, 0 \leqq R \leqq 1$. Then $\left\{A_{R}\left(e^{B t}\right): t \geqq 0\right\}$ is a norm continuous semigroup of completely positive maps if and only if $B$ is of trace class.

Proof. Let $B$ be of trace class. Then we know from $[1,28]$ that $d A_{R}\left(B-B^{*}\right)$ is bounded. Thus using (4.2) it only remains to show that $d A_{R}\left(B+B^{*}\right)$ is bounded. We can find a complete orthonormal set $\left\{f_{n}\right\}$ in $H$, and real scalars $\lambda_{n}, r_{n}$ with $\lambda_{n} \leqq 0,0 \leqq r_{n} \leqq 1, n=1,2, \ldots$, such that

$$
B+B^{*}=\sum \lambda_{n} f_{n} \otimes f_{n}
$$

and $R f_{n}=r_{n} f_{n}$, if $\lambda_{n}<0$. Let $J$ be the unique conjugation on $H$ such that $J f_{n}=f_{n}$. If $p$ is the projection on $\operatorname{Ker}\left(B+B^{*}\right)$, and $T_{t}=\exp \left(B+B^{*}\right) t$, we see that $A_{p R}\left(T_{t}\right)=$ $A_{R}\left(T_{t}\right)$ if $t \geqq 0$. Thus we can assume that $J R=R J$ as well as $J T_{t}=T_{t} J$. Let $E$ be the purification projection on $H \oplus H$ [29]:

$$
E=\left(\begin{array}{cc}
R & R^{1 / 2}(1-R)^{1 / 2} \\
R^{1 / 2}(1-R)^{1 / 2} & 1-R
\end{array}\right)
$$


Then $[J \oplus J, E]=0$. Let $\theta$ be the *-automorphism of $A(H \oplus H)$ given by

$$
\theta a(f)=a((1-E) f)+a^{*}((E)(J \oplus J) f) ; \quad f \in H \oplus H
$$

so that $\omega_{E}=\omega_{0} \circ \theta$. Moreover, since $[J \oplus J, E]=0$ we see that $A_{E}\left(T_{t} \oplus T_{t}\right)=$ $\theta^{-1} A_{0}\left(T_{t} \oplus T_{t}\right) \theta$, for $t \geqq 0$. Hence $\left\{A_{E}\left(T_{t} \oplus T_{t}\right): t \geqq 0\right\}$ is norm continuous by [9]. Thus $A_{R}\left(T_{t}\right)$ is also norm continuous since it is the restriction of $A_{E}\left(T_{t} \oplus T_{t}\right)$ to $A(H)$.

Conversely suppose $d A_{R}(B)$ is bounded. As in [28], let $H_{0}$ be a finite dimensional subspace in $H$ with complete orthonormal set $e_{1}, \ldots, e_{n}, e_{n+1}, \ldots, e_{m}$ such that $B e_{i} \in H_{0}$ for $i=1, \ldots, n$. Then for all $x \in H_{0}$,

$$
B x=\sum_{i=1}^{m}\left\langle B x, e_{i}\right\rangle e_{i}
$$

so that

$$
a(B x) a\left(e_{n+1}\right) \ldots a\left(e_{m}\right)=\sum_{i=1}^{n}\left\langle B x, e_{i}\right\rangle a\left(e_{i}\right) a\left(e_{n+1}\right) \ldots a\left(e_{m}\right)
$$

as $a\left(e_{i}\right) a\left(e_{i}\right)=0$. Thus if $y=a\left(e_{1}\right) \ldots a\left(e_{n}\right)$, and $x=a\left(e_{n+1}\right) \ldots a\left(e_{m}\right)$, we see as in [28] that

$$
\left[d A_{R}(B)(y)\right] x=\sum_{i=1}^{n}\left\langle B e_{i}, e_{i}\right\rangle y x .
$$

Thus

$$
\begin{aligned}
& \sum_{i=1}^{n}\left\langle\left(B+B^{*}\right) e_{i}, e_{i}\right\rangle\left[y x+(y x)^{*}\right] \\
& \quad=\left[d A_{R}(B)(y)\right] x+x^{*} d A_{R}(B)\left(y^{*}\right) .
\end{aligned}
$$

Let $z=y x$; then $z^{2}=0$, since $a\left(e_{i}\right) a\left(e_{i}\right)=0$, moreover $\omega_{0}\left(z^{*} z\right)=0$, and $\omega_{0}\left(z z^{*}\right)=1$ where $\omega_{0}$ is the Fock state. Thus $\left(z+z^{*}\right)^{2}=z z^{*}+z^{*} z$, and $\omega_{0}\left[\left(z+z^{*}\right)^{2}\right]=1$. Whence $\left\|z+z^{*}\right\| \geqq 1$. It now follows from (4.3) that

$$
\left|\sum\left\langle\left(B+B^{*}\right) e_{i}, e_{i}\right\rangle\right| \leqq 2\left\|d A_{R}(B)\right\|<\infty .
$$

Hence since $B+B^{*} \leqq 0$, we see that $B+B^{*}$ is of trace class, with $-\operatorname{tr}\left(B+B^{*}\right) \leqq$ $2\left\|d A_{R}(B)\right\|$. It follows from the first part of the theorem that $d A_{R}\left(B+B^{*}\right)$ is bounded, (as well as $d A_{R}(B)$ by hypothesis). Thus from (4.2) we see that $d A_{R}\left(B-B^{*}\right)$ is bounded and so $B-B^{*}$ is of trace class by $[1,28]$. Hence $B$ is of trace class.

As an application of the theory we have developed, we proceed and take a "Laplacian" which is the square of a quasi-free derivation and consider its perturbation by a quasi-free generator of the type we have constructed already.

Theorem 4.2. Let $h$ be a self-adjoint operator on a hilbert space $H$ and $K$ a bounded dissipative operator on $H$. If $\lambda$ is a real scalar, $0 \leqq \lambda \leqq 1$, then there exists a strongly continuous semigroup of completely positive unital maps on $A(H)$ with infinitesimal generator $L$ such that $A^{1}(H)$ is contained in the domain of $L$ and

$$
L(x)=d A_{\lambda}(i h)^{2}(x)+d A_{\lambda}(K)(x)
$$

for all $x$ in $A^{1}(H)$. 
Proof. Let $\alpha(t)=A\left(e^{\text {iht }}\right)$ denote the strongly continuous group of *-automorphisms of $A(H)$ with infinitesimal generator $i \delta$ say: Let $\left\{f_{t}: t \geqq 0\right\}$ denote the convolution semigroup in $L^{1}(\mathbb{R})$ given by

$$
f_{t}(s)=e^{-s^{2} / t}(\pi t)^{-1 / 2} ; \quad s \in \mathbb{R}, t \in \mathbb{R}^{+} .
$$

Then from semigroup theory [15],

$$
\alpha\left(f_{t}\right)(x)=\int \alpha(s)(x) f_{t}(s) d s, x \in A(H)
$$

defines a strongly continuous semigroup on $A(H)$, with infinitesimal generator $-\delta^{2}$, and which is clearly completely positive [7,8 p.132].

Let $K_{n}$ be a sequence of dissipative trace class operators on $H$, such that $K_{n} \rightarrow K$ strongly, and $\left\|K_{n}\right\| \leqq\|K\|$. Then by Theorem $4.1, S_{n} \equiv d A_{\lambda}\left(K_{N}\right)$ is bounded, and so $-\delta^{2}+S_{n}$ generates a strongly continuous semigroup on $A(H)$, and for each $x$ in $A(H)$

$$
\begin{aligned}
e^{\left(-\delta^{2}+S_{n}\right) t}(x)= & \alpha\left(f_{t}\right)(x)+\int_{0}^{t} \alpha\left(f_{t_{1}}\right) S_{n} \alpha\left(f_{t-t_{1}}\right)(x) d t_{1} \\
& +\ldots \ldots \\
= & \sum_{m=0}^{\infty} A^{n}(m, t)(x)
\end{aligned}
$$

say, where

$$
\begin{aligned}
A^{n}( & m, t)(x) \\
= & \int_{0}^{t} \ldots \int_{0}^{t_{2}} \alpha\left(f_{t_{1}}\right) S_{n} \alpha\left(f_{t_{2}-t_{1}}\right) \ldots S_{n} \alpha\left(f_{t_{m}-t_{m-1}}\right) \\
& \cdot S_{n} \alpha\left(f_{t-t_{m}}\right)(x) d t_{1} \ldots d t_{m} \\
= & \int_{0}^{t} \ldots \int_{0}^{t_{2}} \int_{\mathbb{R}^{m}} e^{i \delta_{s_{1}}} S_{n} e^{i \delta s_{2}} \ldots S_{n} e^{i \delta s_{m}}(x) \\
& \cdot f_{t_{1}}\left(s_{1}\right) f_{t_{2}-t_{1}}\left(s_{2}\right) \ldots f_{t-t_{m}}\left(s_{m}\right) d s_{1} \ldots d s_{m} d t_{1} \ldots d t_{m}
\end{aligned}
$$

For all $f_{1}, \ldots, f_{p}$ in $H$ we have

$$
\left\|: a^{\#}\left(f_{1}\right) \ldots a^{\#}\left(f_{p}\right):_{\lambda}\right\| \leqq 2^{p}\left\|f_{1}\right\| \ldots\left\|f_{p}\right\|
$$

Let $y=: a^{\#}\left(g_{1}\right) \ldots a^{\#}\left(g_{p}\right):_{\lambda}$, where $g_{1}, \ldots, g_{p}$ are unit vectors in $H$. Then from (4.1), (4.5) and (4.6) we see that

$$
\begin{aligned}
\left\|A^{n}(m, t)(y)\right\| & \leqq 2^{p} p^{m}\left\|K_{n}\right\|^{m} t^{m} / m \\
& \leqq 2^{p}(p\|K\| t)^{m} / m
\end{aligned}
$$

It is also clear from (4.5) that $\lim A^{n}(m, t)(y)$ exists. Hence $\lim e^{\left(-\delta^{2}+S_{n}\right) t}(x)$ exists for all $t \geqq 0$, in $A(H)$ and defines a strongly continuous semigroup of completely positive unital maps, whose generator $L$ satisfies (4.4).

\section{Appendix}

Proposition A.1. Let $T: H \rightarrow K$ be a contraction between Hilbert spaces $H$ and 
$K$, which intertwines with positive contractions $R$ and $S$ on $H$ and $K$ respectively. Then for all $f(1), \ldots, f(m), g(1), \ldots, g(n)$ in $H$, we have:

$$
\begin{aligned}
& A_{R}(T)\left[: a^{*}(f(1)) \ldots a^{*}(f(m)) a(g(n)) \ldots a(g(1)):_{R}\right] \\
& \quad=\quad: a^{*}(T f(1)) \ldots a^{*}(T f(m)) a(T g(n)) \ldots a(T g(1)):_{S}
\end{aligned}
$$

Notation. If $\sigma=\left(\sigma_{1}, \ldots, \sigma_{r}\right)$ is a sequence of positive integers, we let $|\sigma|$ denote its length $r$, and $\{\sigma\}$ its underlying set $\left\{\sigma_{i}\right\}$. If $\sigma^{i}=\left(\sigma_{1}^{i}, \ldots, \sigma_{r_{i}}^{i}\right) r_{i}=\left|\sigma^{i}\right|$, is a finite number of sequences, we let $\left(\sigma^{1}, \ldots, \sigma^{p}\right)$ denote the sequence $\left(\sigma_{1}^{1}, \ldots, \sigma_{r_{1}}^{1}, \sigma_{1}^{2}, \ldots\right.$, $\left.\sigma_{r_{2}}^{2}, \ldots, \sigma_{1}^{p}, \ldots, \sigma_{r_{p}}^{p}\right)$. If $\left\{\left(\sigma^{1}, \ldots, \sigma^{p}\right)\right\}=\left\{j_{1}>\ldots>j_{q}\right\}$, we let $\operatorname{sgn}\left(\sigma^{1}, \ldots, \sigma^{p}\right)$ denote the signature of the permutation $\left(j_{1}, \ldots, j_{q}\right) \rightarrow\left(\sigma^{1}, \ldots, \sigma^{p}\right)$ when it exists. If $\sigma=\left(\sigma_{i}\right)$ is a finite sequence, with $1 \leqq \sigma_{i} \leqq n, r=|\sigma|$, we let

$$
\begin{aligned}
& a(g(\sigma))=a\left(g\left(\sigma_{1}\right)\right) \ldots \ldots a\left(g\left(\sigma_{r}\right)\right) \\
& a(T g(\sigma))=a\left(T g\left(\sigma_{1}\right)\right) \ldots \ldots a\left(T g\left(\sigma_{r}\right)\right) \\
& a^{*}(g(\sigma))=[a(g(\sigma))]^{*}, a^{*}(T g(\sigma))=[a(T g(\sigma))]^{*}
\end{aligned}
$$

We let $X_{n}$ denote the set of all decreasing sequences $\sigma=\left(\sigma_{i}\right), \sigma_{1}>\sigma_{2}>\ldots>\sigma_{r}$, with $r=|\sigma| \leqq n$. If $\sigma, \rho^{1}, \ldots, \rho^{p} \in X_{n}$, with $\{\sigma\}=\bigcup_{j=1}^{p}\left\{\rho^{j}\right\}$ and $\left\{\rho^{i}\right\} \cap\left\{\rho^{j}\right\}=\Phi$, if $i \neq j$, we write $\sigma=\rho^{1}+\ldots+\rho^{p}$.

Lemma A.2. If $D=\left(1-T^{*} T\right)^{1 / 2}$, we have:

$$
\begin{aligned}
& \omega_{T^{*} \mathrm{~S} T}\left[a^{*}(f(m, \ldots, 1)) a(g(n, \ldots, 1))\right] \\
& \quad=\sum \eta \omega_{R}\left[a^{*}(f(\sigma)) a(g(\rho))\right] \omega_{D^{2} R}\left[a^{*}\left(f\left(\sigma^{\prime}\right)\right) a\left(g\left(\rho^{\prime}\right)\right)\right]
\end{aligned}
$$

where the summation extends over all $\sigma, \sigma^{\prime}$ in $X_{m}, \rho, \rho^{\prime}$ in $X_{n}$, with $\sigma+\sigma^{\prime}=(m, \ldots, 1)$, $\rho+\rho^{\prime}=(n, \ldots, 1)$, and

$$
\eta=(-1)^{\left|\sigma^{\prime}\right|} \operatorname{sgn}\left(\rho^{\prime}, \rho\right) \operatorname{sgn}\left(\sigma^{\prime}, \sigma\right) \text {. }
$$

Proof. By induction and the relation

$$
\omega_{T^{*} S T}\left[a^{*}(f) a(g)\right]=\omega_{R}\left[a^{*}(f) a(g)\right]-\omega_{D^{2} R}\left[a^{*}(f) a(g)\right]
$$

valid for all $f$ and $g$ in $H$, (which is the case $m=n=1$ ).

Proof of Proposition A.1. We have

$$
\begin{aligned}
& : a^{*}\left(f(m, \ldots, 1) a(g(n, \ldots, 1)):_{R}\right. \\
& \quad=\sum \varepsilon a^{*}\left(f\left(\alpha_{0}\right)\right) a\left(g\left(\beta_{0}\right)\right) \omega_{R}\left[a^{*}(f(\alpha)) a(g(\beta))\right]
\end{aligned}
$$

where the summation extends over all $\alpha, \alpha_{0}$ in $X_{m}, \beta, \beta_{0}$ in $X_{n}$, with $\alpha+\alpha_{0}=$ $(\mathrm{m}, \ldots, 1), \beta+\beta_{0}=(n, \ldots, 1)$, and

$$
\varepsilon=(-1)^{\left|\alpha_{0}\right|\left|\beta_{0}\right|} \operatorname{sgn}\left(\alpha, \alpha_{0}\right) \operatorname{sgn}\left(\beta, \beta_{0}\right) .
$$

Moreover for all $\alpha_{0} \in X_{m}, \beta_{0} \in X_{n}$ :

$$
\begin{aligned}
& A_{R}(T)\left[a^{*}\left(f\left(\alpha_{0}\right)\right) a\left(g\left(\beta_{0}\right)\right)\right] \\
& \quad=\sum \theta a^{*}\left(T f\left(\alpha^{\prime \prime}\right) a\left(T g\left(\beta^{\prime \prime}\right)\right) \omega_{D^{2} R}\left[a\left(f\left(\alpha^{\prime}\right)\right) a\left(g\left(\beta^{\prime}\right)\right)\right]\right.
\end{aligned}
$$


where the summation extends over all $\alpha^{\prime}, \alpha^{\prime \prime}$ in $X_{m}, \beta^{\prime}, \beta^{\prime \prime}$ in $X_{n}$ such that $\alpha^{\prime}+\alpha^{\prime \prime}=$ $\alpha_{0}, \beta^{\prime}+\beta^{\prime \prime}=\beta_{0}$, and

$$
\theta=(-1)^{\left|\alpha^{\prime}\right|\left|\alpha^{\prime \prime}\right|+\left|\beta^{\prime}\right|\left|\beta^{\prime \prime}\right|} \operatorname{sgn}\left(\alpha^{\prime}, \alpha^{\prime \prime}\right) \operatorname{sgn}\left(\beta^{\prime}, \beta^{\prime \prime}\right) \text {. }
$$

Thus from (A.1) and (A.2), we have:

$$
\begin{aligned}
& A_{R}(T)\left[: a^{*}(f(m, \ldots, 1)) a(g(n, \ldots, 1)):_{R}\right] \\
& \quad=\sum \eta a^{*}\left(T f\left(\alpha^{\prime \prime}\right) a\left(T g\left(\beta^{\prime \prime}\right)\right) \omega_{D^{2} R}\left[a^{*}\left(f\left(\alpha^{\prime}\right)\right) a\left(g\left(\beta^{\prime}\right)\right)\right] \omega_{R}\left[a^{*}(f(\alpha)) a(g(\beta))\right]\right.
\end{aligned}
$$

where the summation extends over all $\alpha^{\prime \prime}, \alpha^{\prime}, \alpha$ in $X_{m}, \beta^{\prime \prime}, \beta^{\prime}, \beta$ in $X_{n}$ such that $\alpha^{\prime \prime}+\alpha^{\prime}+\alpha=(m, \ldots, 1), \beta^{\prime \prime}+\beta^{\prime}+\beta=(n, \ldots, 1)$ and

$$
\begin{aligned}
\eta & =(-1)^{\left|\alpha^{\prime \prime}\right|\left|\beta^{\prime \prime}\right|+\left|\beta^{\prime}\right|} \operatorname{sgn}\left(\alpha, \alpha^{\prime}, \alpha^{\prime \prime}\right) \operatorname{sgn}\left(\beta, \beta^{\prime}, \beta^{\prime \prime}\right) ; \\
& =\sum \varepsilon a^{*}\left(T f\left(\alpha^{\prime \prime}\right)\right) a\left(T g\left(\beta^{\prime \prime}\right)\right) \omega_{T^{*} S T}\left[a^{*}(f(\delta)) a(g(\lambda))\right]
\end{aligned}
$$

using Lemma A.2., where the summation extends over all $\alpha^{\prime \prime}, \delta$ in $X_{m}, \beta^{\prime \prime}, \lambda$ in $X_{n}$, such that $\alpha^{\prime \prime}+\delta=(m, \ldots, 1), \beta^{\prime \prime}+\lambda=(n, \ldots, 1)$, and

$$
\varepsilon=(-1)^{\left|\alpha^{\prime \prime}\right|\left|\beta^{\prime \prime}\right|} \operatorname{sgn}\left(\delta, \alpha^{\prime \prime}\right) \operatorname{sgn}\left(\lambda, \beta^{\prime \prime}\right) \text {. }
$$

The Proposition follows.

Acknowledgements. This work was commenced at the University of Oslo, and completed whilst the author was visiting the University of California, Los Angeles. The author would like to express his sincere thanks to E. St $\phi$ rmer at Oslo and H. A. Dye and M. Takesaki at UCLA for their warm hospitality. He is also grateful to E. B. Davies, L.-E. Lundberg and M. Takesaki for stimulating discussions.

\section{References}

1. Araki, H. : Publ. RIMS Kyoto Univ. 6, 385-442 (1971)

2. Araki, H., Wyss, W. : Helv. Phys. Acta. 37, 136-159 (1964)

3. Arveson, W. B. : Acta Math. 123, 141-224 (1969)

4. Balslev, E., Verbeure, A. : Commun. Math. Phys. 7, 55-76 (1968)

5. Bratteli, O., Robinson, D. W. : Ann. Inst. H. Poincaré 25, 139-164 (1976)

6. Cooper, J. L. B. : Ann. of Math. 48, 827-842 (1947)

7. Davies, E. B. : Z. Wahrschein. 23, 261-273 (1972)

8. Davies, E. B. : Quantum theory of open systems. Academic Press 1976

9. Davies, E. B. : Commun. Math. Phys. 55, 231-258 (1977)

10. Davies, E. B. : J. Lon. Math. Soc. 17, 330-338 (1978)

11. Davies, E. B. : Generators of dynamical semigroups. Preprint, Oxford

12. Dell'Antonio, G. F. : Commun. Math. Phys. 9, 81-117 (1968)

13. Demoen, B., Vanheuverzwijn, P., Verbeure, A. : Lett. Math. Phys. 2, 161-166 (1977)

14. Demoen, B., Vanheuverswijn, P., Verbeure, A.: Completely positive quasi-free maps on the CCR algebra. Preprint, Leuven

15. Dunford, N., Schwartz, J. T. : Linear operators I. New York: Interscience (1958)

16. Emch, G. G., Albeverio, S., Eckmann, J.-P. : Rep. Math. Phys. 13, $73-85$ (1978)

17. Evans, D. E. : Commun. Math. Phys. 48, 15-22 (1976)

18. Evans, D. E., Lewis, J. T. : Commun. Math. Phys. 50, 219-227 (1976)

19. Evans, D. E., Lewis, J. T. : J. Funct. Anal. 26, 369-377 (1977)

20. Evans, D. E., Lewis, J. T. : Dilations of irreversible evolutions in algebraic quantum theory. Comm. Dub. Inst. Adv. Studies, Ser A, 24, 1977

21. Haag, R., Hugenkoltz, N. M., Winnik, M. : Commun. Math. Phys. 5, 215-236 (1967) 
22. Halmos, P. R. : A hilbert space problem book. Princeton: Van Nostrand 1967

23. Hugenholtz, N. M., Kadison, R. V. : Commun. Math. Phys. 43, 181-197 (1975)

24. Ingarden, R. S., Kossakowski, A. : Ann. Physics 89, 451-485 (1975)

25. Kossakowski, A., Frigerio, A., Gorini, V., Verri, M. : Commun. Math. Phys. 57, 97-110 (1977)

26. Lindblad, G. : Rep. Math. Phys. 10, 393-406 (1976)

27. Manuceau, J., Rocca, F., Testard, D. : Commun. Math. Phys. 12, 43-57 (1969)

28. McGovern, R.J. : J. Funct. Anal. 26, 89-101 (1977)

29. Powers, R. T., St $\phi$ rmer, E. : Commun. Math. Phys. 16, 1-33 (1970)

30. Rocca, F., Siruge, M., Testard, D. : Commun. Math. Phys. 13, 317-334 (1970)

31. Schrader, R., Uhlenbrock, D. A. : J. Funct. Anal. 18, 369-413 (1975)

32. Shale, D., Stinespring, W. F. : Ann. Math. 80, 365-381 (1964)

33. Nagy, Sz., B. Foias, C. : Harmonic analysis of operators on Hilbert space. Amsterdam: North Holland 1970

34. Takesaki, M. : Tomita's theory of modular hilbert algebras and its applications. Lecture notes in math. Vol. 128. Berlin, Heidelberg, New York: Springer 1970

35. Takesaki, M. : J. Funct. Anal. 9, 306-321 (1972)

Communicated by $\mathrm{H}$. Araki

Received June 26, 1978; in revised form May 21, 1979 